\title{
Band-Pass Continuous-Time Delta-Sigma Modulators Employing LWR Resonators
}

\author{
Mohammad Javidan \\ Department of Signal Processing and Electronic Systems \\ SUPELEC \\ F91192 GIF/YVETTE, France \\ Mohammad.javidan@supelec.fr
}

\author{
Philippe Bénabès, IEEE Member \\ Department of Signal Processing and Electronic Systems \\ SUPELEC \\ F91192 GIF/YVETTE, France \\ philippe.benabes@supelec.fr
}

Abstract- A new topology and design method for continuous-time (CT) band-pass delta-sigma modulators is presented in this paper. The proposed modulator employs a Lamb Wave Resonator (LWR) possessing high Q-factor. This kind of resonator presents many advantages comparing with the $L C$ or $G_{m}-C$ resonators. Furthermore, the proposed topology is entirely compatible with the LWR transfer functions. This method facilitates the design of band-pass delta-sigma modulators in a wide range of central frequencies $\left(f_{c}\right)$.

\section{INTRODUCTION}

Band-pass delta-sigma modulators are widely used in telecommunication and mobile technologies where the needs for low-power, high-resolution and small-size are of critical importance. Recently, several CT delta-sigma modulators based on $G_{m}-c$ or $L C$ resonators have been reported. Their major advantage is compatibility with all the Integrated Circuit (IC) implementation technologies [1]. However, it is known that these modulators suffer from performance degradation for a number of reasons. First of all, in narrow band digitization, the non-idealities of constituent $\mathrm{G}_{\mathrm{m}} \mathrm{c}$ or $\mathrm{LC}$ resonators are the limiting factors. Secondly it is difficult to realize the resonators with high Q-factor due to the parasitic loss, especially at high frequencies. Although Q-enhancement circuits can be employed, the linearity of the resonator will be deteriorated. In addition the resonant frequency of the resonator is sensitive to process and temperature variations. Consequently it is still difficult to achieve high accuracy, especially in the case of narrowband digitization [2]. In order to improve the actual performance of the CT delta-sigma modulator, the need of an accurate resonator with high-Q factor is critical.

Recent progress has made the integration of micromechanical devices with CMOS circuits on a single chip possible. Moreover, it is well known that the electromechanical resonators are accurate resonators and possess high-Q factors [3]. In this research, micro-mechanical resonators, especially Lamb Wave Resonators are applied to design the delta-sigma modulator.

\section{LAMB WAVE RESONATOR (LWR)}

The structure of Lamb Wave Resonator (LWR) which is similar to Acoustic Wave resonator (SAW) and Film Bulk Acoustic Resonator (FBAR), is composed of a piezoelectric layer sandwiched between two thin electrodes and placed on a membrane (Fig. 1). However, FBAR operates using vertical wave propagation; the LWR employs lateral wave propagation. Indeed, in LWR, the resonance appears when the length $\mathrm{L}$ is proportional to half wavelength. Changing the resonance frequency, while keeping standard lateral dimension, is possible through specific electrode designs [4].

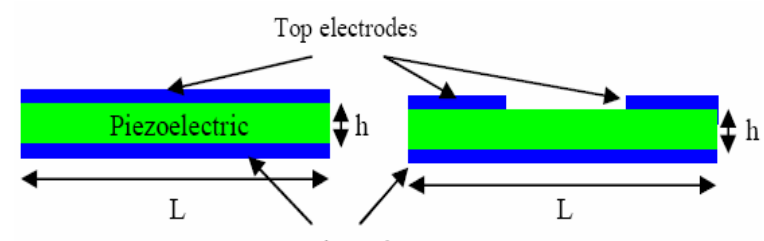

(a) Bottom electrodes

(b)

Fig. 1. Lamb wave resonator working on (a) fundamental (b) third harmonic

The advantages of LWR can be listed as: 1- Accurate central frequency without the need for tuning. 2- High loaded Qfactor above 500 that can be easily attained. 3- Wide available resonant frequency range up to Gigahertz. 4- High temperature stability.

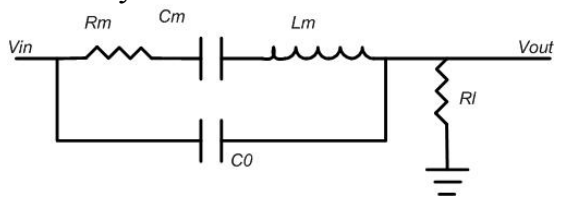

Fig. 2. Model of one-port LWR resonator

Fig. 2 shows a model used for most of one-port micromechanical resonators which can be connected to a resistive load $R_{L}$. This model can be also a modeler of the LWR. $R_{m}$, $C_{m}$ and $L_{m}$ are the motional resistance, capacitance and inductance, respectively. $C_{0}$ is the inherent static capacitance between the two terminals. These parameters could be found through the resonance and anti-resonance frequencies, which are the results of a finite element simulation [4]. Equation (1) is the transfer function of the LWR resonator.

$$
H_{L W R}=\frac{s\left(s^{2}+\frac{R_{m}}{L_{m}} s+\frac{C_{0}+C_{m}}{L_{m} C_{m} C_{0}}\right)}{S^{3}+\left(\frac{R_{m}}{L_{m}}+\frac{1}{R_{L} C_{0}}\right) s^{2}+\left(\frac{\left(R_{m}+R_{L}\right) C_{m}+R_{L}+C_{0}}{L_{m} C_{m} R_{L} C_{0}}\right) s+\frac{1}{L_{m} C_{m} R_{L} C_{0}}}
$$

Regarding this model, like the other micro-mechanical resonators, the LWR gives a resonance and anti-resonance frequencies. The resonance frequency $\left(\omega_{s}\right)$, anti-resonance 
frequency $\left(\omega_{p}\right)$ and Q-factor $(Q)$ of the resonator can be calculated, respectively, as follow:

$$
\omega_{s}=\frac{1}{\sqrt{L_{m} C_{m}}} \quad \omega_{p}=\sqrt{\frac{C_{m}+C_{0}}{L_{m} C_{m} C_{0}}} \quad Q=\frac{1}{R_{m}} \sqrt{\frac{L_{m}}{C_{m}}}
$$

$H_{l w r}$ does not have an ideal second-order resonator transfer function and moreover, it is passive and has an insertion loss. These issues lead us to propose a new resonator structure (Fig. 3), where $x$ referents the LWR.

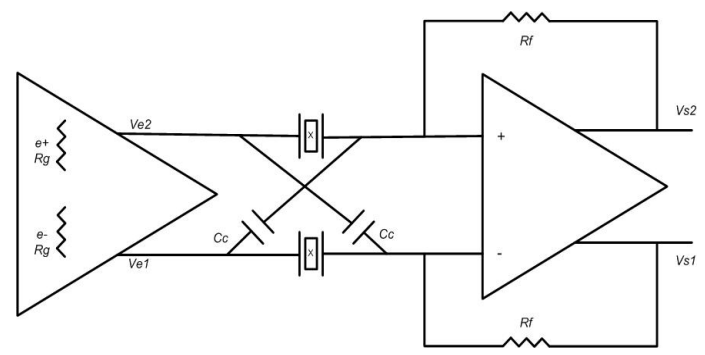

Fig. 3. Proposed resonator topology to anti-resonance cancelation

The major advantage of this structure is the anti-resonance cancelation. Two capacitive paths $\left(C_{c}\right)$ are added and a differential signal is used to drive the resonator and $C_{c}$. These paths act effectively as a negative capacitance. If $C_{c}$ is made equal to $C_{0}$, the effect of $C_{0}$ can be completely cancelled. Additionally the insertion loss of the resonator can be compensated by the gain stage. Supposing an ideal amplifier $\left(A_{v}>>1 /\left(R_{m} C_{m}\right) \& R_{g}=0\right)$ the result of the transfer function for resonator topology can be expressed as follow.

$$
H_{r e s}=\frac{\left(R_{f} c_{m}\right) s}{1+\left(R_{m} C_{m}\right) s+L C s^{2}}
$$

$H_{\text {res }}$ can be regarded as an ideal second-order resonator. Consequently this topology based on the LWR, seems to be a prefect candidate for the delta-sigma modulator filter and will be used all over this study.

\section{CONTINUOUS-TIME MODULATOR SYNTHESIS}

Before the illustration, it should be noted that our goal is to design a large band delta-sigma modulator which work at a central frequency between $0.2 f_{s}$ and $0.3 f_{s}$ where $f_{s}$ is the sampling frequency. Consequently the proposed method is reliable for central frequencies between $0.2 f_{s}$ and $0.3 f_{s}$.

Several structures have been reported to synthesise a CT delta-sigma global filter. The most famous of them are monoboucle structure [5] and multi-boucle structure [6].

The filter transfer function of the mono-boucle structure $\left(\frac{b_{1} s+b_{0}}{s^{2}+a_{1} s+a_{2}}\right)$ benefits from the $b_{0}$ term which is put to use as a modifiable parameter to synthesise and as a result it can not be eliminated. So this transfer function can exclusively be realized using Gm-c or LC resonators due to the existence of this term. On the other hand, due to the several modifiable parameters, the multi-boucle topology is a flexible structure that can support all type of transfer function. However the number of modifiable parameters complicates the calculation and the circuit implementation. In this paper a new topology, using only 'pure resonator' is presented (Fig.4). There are two major advantages for this structure: 1- The filter transfer function of this structure is compatible with the LWR. 2- This topology employs a minimum number of modifiable parameters.

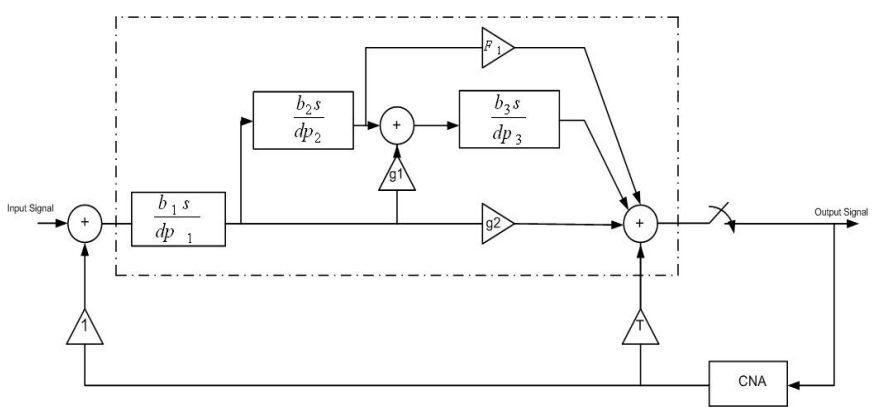

Fig. 4. Proposed topology for a Continuous-time $6^{\text {th }}$ order delta-sigma

The design of the delta-sigma modulator using the topology of Fig. 4 will be explained in four steps.

\section{A. Global Filter Design}

The best way to design a CT delta-sigma modulator is to start from a DT delta-sigma modulator filter transfer function, which satisfies all the demand in term of resolution and stability. Then, the transformation expressed in (4) will be used in order to achieve its CT delta-sigma filter counterpart.

$$
F(Z)=\left(1-Z^{-1}\right) Z_{T}\left\{L^{-1}\left[\frac{G(s) B(s)}{s}\right]\right\}-\sum_{k} a_{k} z^{-k}
$$

Where $L^{-1}$ denotes the inverse Laplace transform, $Z_{T}$ the ztransform at sampling period $\left(T_{s}\right)$ and $B(s)$ denotes delay and non-ideality part of DAC and ADC functionality. In this approach, we use standard tools available in symbolic calculation programs such as Laplace and z-transform.

This method which is vastly explained in [5] is almost preferred due to its facility and the low cost of the simulation time in DT domain.

\section{B. DAC Delay Optimization}

The global filter transfer function of Fig.4 (dotted line) is calculated in (5). $d p_{1}, d p_{2}$ and $d p_{3}$, the enumerators of the resonators, are second order polynomials $\left(a_{0} s^{2}+a_{1} s+a_{2}\right)$.

$$
H_{\text {Filter }}=\frac{b_{1} s}{d p_{1}}\left[\left(F_{1}+\frac{b_{3} s}{d p_{3}}\right) \frac{b_{2} s}{d p_{2}}+g_{2}+g_{1} \frac{b_{3}}{d p_{3}}\right]
$$

Multiplying $b_{l} s$ at all the expression proves that there exists no " $s^{0}$ "term in the numerator of $H_{\text {Filter. }}$. Consequently, regarding the form of $H_{\text {Filter }}$, it's really essential that the numerator of $G(s)$ has also no " $s$ " term. It means that the $G(s)$ calculated by (4) is usually, out of form unless the DAC delay is correctly settled. However, the DAC delay can be a help to solve this issue.

Fig. 5 shows the coefficient value of " $s$ " term variation of the $G(s)$ numerator versus DAC delay for several central frequencies for a sixth order delta-sigma modulator. As it is shown, this variation is almost linear versus DAC delay and consequently it is possible to calculate a DAC delay for which the " $s$ " term is equal to zero. We proposed an 
optimization method on DAC delay to formulate $G(s)$. In this method, a quasi-linear function is calculated to find this DAC delay without simulation.

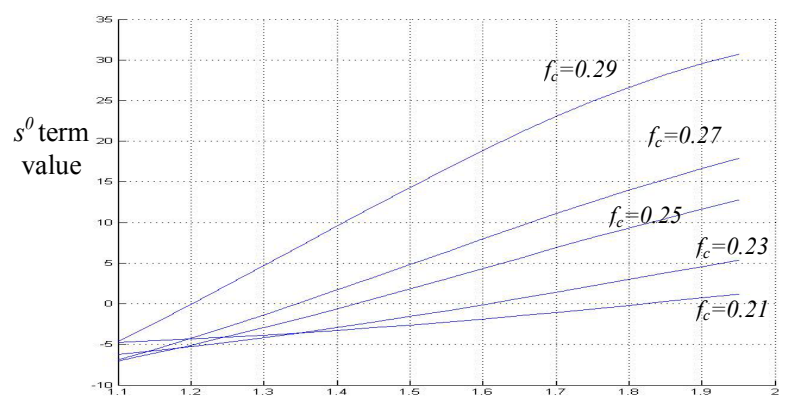

Fig. 5. $s^{0}$ term variation versus DAC delay delay

\section{Synthesis Method}

To realize $G(s)$, we require equating the numerator $\left(N_{G(s)}\right)$ and the enumerator $\left(E_{G(s)}\right)$ of $G(s)$ by the numerator $\left(N_{\text {Filter }}\right)$ and the enumerator $\left(E_{\text {Filter }}\right)$ of $(5)$, respectively. This condition leads to constitute a non-linear system of equations which is given by (6). $n$ and $m$ are respectively, the degree of numerator and enumerator of $G(s)$.

$$
\left\{\begin{array}{l}
\sum_{j=1}^{m} E_{G(s)_{m}} s^{m}=\sum_{j=1}^{m} E_{\text {Filter } m} s^{m} \\
\sum_{i=1}^{n} N_{G(s)_{n}} s^{n}=\sum_{i=1}^{i=n} N_{\text {Filter } n} s^{n}
\end{array}\right.
$$

In order to calculate the unknown parameters, we need to simultaneously solve the two parts of (6). This system of equation should provide a sufficient number of equations for calculating all unknown coefficients. Regarding the numerator's order of $G(s)$ and $H_{\text {filter, }} F_{l}$ is expressed in (7) in order to grant the sufficient number of equations.

$$
F_{1}=g_{3}+g_{4} \frac{1}{s}
$$

As a result the vector of unknown parameters will be: $\left[b_{1}, b_{2}\right.$, $\left.b_{3}, g_{1}, g_{2}, g_{3}, g_{4}\right]$. The actual mathematical solver routines which are usually the standard algorithm as those available in the MATLAB optimization toolbox can be used. Here we propose to use the Newton's method to solve the system of equations. Newton's method computes the equation and its Jacobian matrix for each iteration, in order to find a step by solving the system of linear equations. The Jacobian matrix of (6) is calculated in (8). This method only works if the initial value vector $\left[b_{10}, b_{20}, b_{30}, g_{10}, g_{20}, g_{30}, g_{40}\right]$ is close enough to the true zero. Consequently it is important to study the Jacobian matrix of the non-linear equation to find the best start point.

$$
\left[\begin{array}{cccc}
\frac{\sigma\left(E_{G(s) 1}-E_{\text {filter } 1}\right)}{\sigma b_{1}} & \ldots & \ldots & \frac{\sigma\left(E_{G(s) 1}-E_{\text {filter } 1}\right)}{\sigma g_{4}} \\
\cdot & \ldots & \ldots & \cdot \\
\cdot & \ldots & \ldots & \cdot \\
\frac{\sigma\left(E_{G(s) m}-E_{\text {filterm }}\right)}{\sigma b_{1}} & \ldots & \ldots & \frac{\sigma\left(E_{G(s) m}-E_{\text {filterm }}\right)}{\sigma g_{4}}
\end{array}\right]
$$

The calculation results of (6) shows that for the central frequency between $0.2 f_{s}$ and $0.3 f_{s}$ the value of $g_{4}$ is almost equal to zero; so it can be neglected to simplify the realizing and $F_{1}$ can be replaced by $g_{3}$ with no effect on performance. Table. 1 shows the value of $g_{4}$ for several frequencies.

Table1. $g_{4}$ variation versus central frequency

\begin{tabular}{|l|l|l|l|l|}
\hline Central frequency & $0.21 f_{s}$ & $0.24 f_{s}$ & $0.27 f_{s}$ & $0.3 f_{s}$ \\
\hline$g_{4}$ value & 0.02 & 0.032 & 0.058 & 0.082 \\
\hline
\end{tabular}

D. Stability Analysis

CT topologies are sensitive to analog parameters. Large mismatches in analog parameters may lead to performance degradation or even instability. We propose to analyze stability using the modulus margin. Modulus margin is a number which can be a comprehensible reference to compare different circuits' stability and sensitivity. Therefore, the modulus margin should be as large as possible.

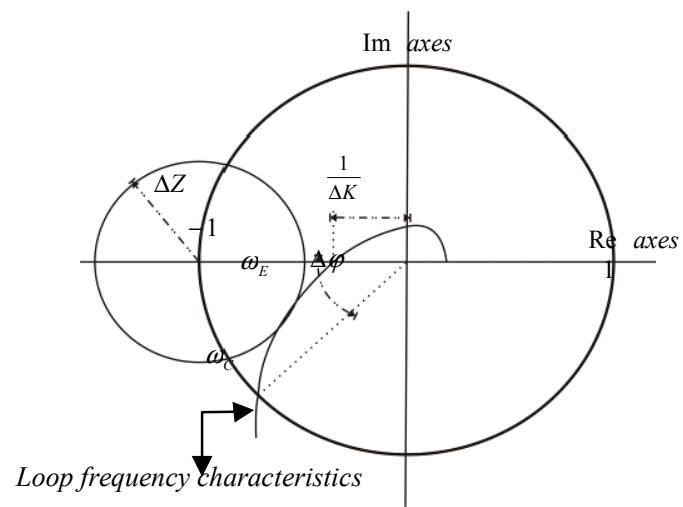

Fig. 6. Modulus $(\Delta Z)$, gain $(\Delta K)$ and phase $(\Delta \varphi)$ margins for a loop frequency characteristics

Modulus, gain and phase margin indicators correspond to different geometrical terms, characterizing the distance between the Nyquist plot of the open-loop and the critical point $\left[-1, j_{0}\right]$. As it is shown in Fig. 6, the modulus margin $(\Delta z)$ is defined as the radius of the circle centered in $\left[-1, j_{0}\right]$ and tangent to the Nyquist plot of the filter [7].

The definition of the vector connecting the critical point, with the Nyquist plot, results in:

$$
\begin{aligned}
& \Delta z=\min \{(1+\operatorname{LFT}(j \omega))\}=\min \left\{\left|s^{-1}(j \omega)\right|\right\} \Rightarrow \\
& \Delta z=(\max \{|s(j \omega)|\})^{-1}=\left(\|s(j \omega)\|_{\infty}\right)^{-1}
\end{aligned}
$$

LFT is the Loop Filter Transfer function of the system. In another word the modulus margin is equal to the inverse of the modulus of the sensitivity function $s(j \omega)$. So modulus margin covers the gain and phase margins information. It is preferable to be as little sensitive as possible to analog parameters. This margin is substantial and is counted as one of the most important parameters in the design of CT modulators.

This design procedure is illustrated in the following chapter through the following example.

\section{DESIGN EXAMPLE AND SimUlation RESUltS}

Let us consider a sixth order delta-sigma modulator working at a central frequency equal to $0.22 f_{s}$. The OSR is equal to 64 and the Q-factor is chosen equal to the realistic value of 900 . 
The DAC delay value, for which the coefficient of " $s$ " is equal to zero, is calculated out of the optimization method on DAC delay and is approximately equal to $1.71 T_{s}$; where $T_{s}$ is the sampling period. So, through (4) the global filter transfer function can be calculated as follow

$G(s)=\frac{-1.6837 \mathrm{~s}^{5}-0.76101 \mathrm{~s}^{4}-6.6006 \mathrm{~s}^{3}-1.4711 \mathrm{~s}^{2}-1.4711 \mathrm{~s} \quad(10)}{\mathrm{s}^{6}+0.0046077 \mathrm{~s}^{5}+5.733 \mathrm{~s}^{4}+0.017608 \mathrm{~s}^{3}+10.953 \mathrm{~s}^{2}+0.016818 \mathrm{~s}+6.9735}$

Afterwards using the system of non-linear equations (6), the unknown parameters of Fig.4 has been found.

$$
\left\{\begin{array}{l}
b_{1}=1.00 \quad b_{2}=0.6612 \quad b_{3}=0.20 \\
g_{1}=1.3650 \quad g_{2}=1.6829 \quad g_{3}=0.7273 \quad g_{4}=0.0211 \\
d p_{1}=s^{2}+0.0014924 s+1.9108 \\
d p_{2}=s^{2}+0.0014619 s+1.8333 \\
d p_{3}=s^{2}+0.001523 s+1.9898
\end{array}\right.
$$

This procedure can be applied easily to design the deltasigma modulator for different central frequencies.

Fig.7 shows the Noise Transfer Function (NTF) of the topology of Fig.4 for three central frequencies. $\left(f_{c}=0.22 f_{s}\right.$, $\left.f_{c}=0.25 f_{s}, f_{c}=0.28 f_{s}\right)$. The DAC delay value is respectively equal to $1.71 T_{s}, 1.43 T_{s}$ and $1.2 T_{s}$ and it should be noted that $\mathrm{g}_{4}$ is neglected all over the simulations.

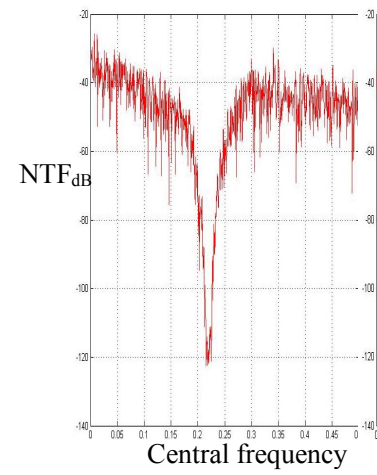

(a)

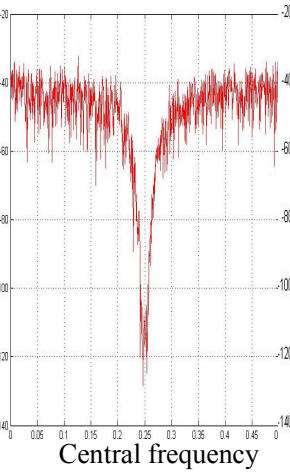

(b)

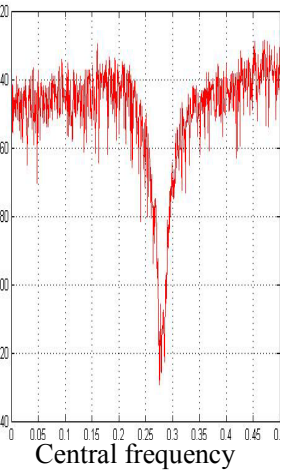

(c)
Fig. 7. Noise Transfer Function for three central frequencies $\left(f_{c}\right)$. (a): $f_{c}=0.22$ (b): $f_{c}=0.25$ (c) $: f_{c}=0.28$

In order to analyze the stability through the modulus margin, first, the loop filter transfer function (LFT) should be calculated. However, due to the sample and hold function at the ADC input, the global transfer function of the modulator cannot be expressed in continuous-time.

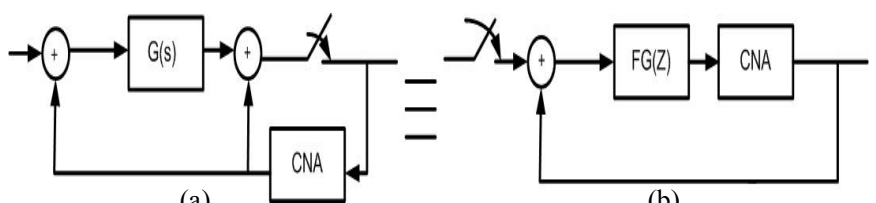

(a)

(b)

Fig. 8. (a): CT topology (b): DT counterpart of (a), the sampler is out of the loop.

The proposed solution in this paper uses a transformation of CT modulator to its DT equivalent (Fig.8). Afterward the modulus margin can be calculated through (9) and $F_{G}(Z)$.
With the intention of benchmarking the design, the result of stability analysis (Fig. 9) is compared with that of a Schereier's modulator topology [1].

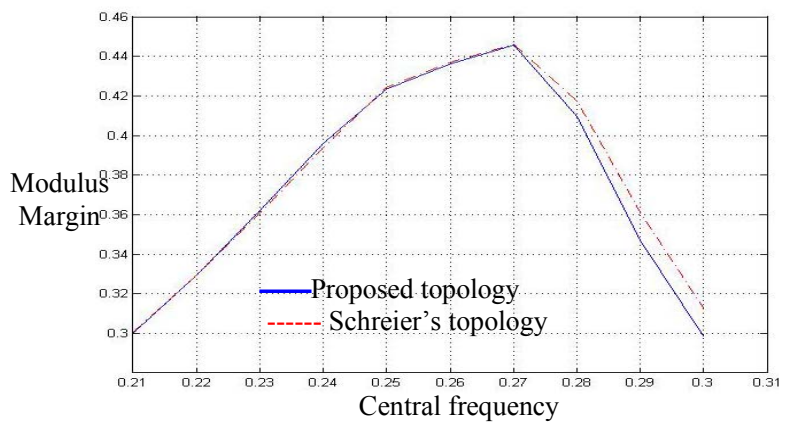

Fig. 9. Modulus margin for the proposed topology and the Schreier's topology

The results show that the performance of this modulator matches perfectly that of a $6^{\text {th }}$ order delta-sigma modulator in spite of the approximation due to removing $g_{4}$. It should be mentioned that this structure can easily be extended to orders higher than three, by creating three gain stages for each added resonator.

\section{CONCLUSION}

In this paper, a new topology and method to synthesize the band-pass continuous-time delta-sigma modulators using the LWR was briefly presented. Employing LWR resonators is a help to avoid performance degradation due to the low Qfactor of the $\mathrm{LC}$ or $\mathrm{G}_{\mathrm{m}}-\mathrm{C}$ resonators. LWR has the advantage of high Q-factor, wide resonant frequency range and accurate resonant frequency. With the proposed anti-resonance cancellation method, it seems to be the prefect candidate for delta-sigma modulator's resonator. Another advantage of this method is the facility of calculations and implementation of the topology due to lower number of components comparing with the other topologies. Finally a stability study, based on modulus margin is presented which alleviates the comparison of stability and sensitivity of different topologies.

\section{REFERENCES}

[1] R. Schreier, B. Zhang, "Delta-Sigma Modulators Employing Continuous-Time Circuitry", IEEE Transactions on Circuits and Systems, Vol. 43, Pp. 324-332, April 1996

[2] R. Yu, Y.P. Xu, "Band-pass Sigma-Delta Modulator Employing SAW Resonator as Loop Filter", IEEE Transactions on Circuits and Systems, Regular Papers, Vol. 54, No. 4, April 2007

[3] X. Wang, Y.P Xu, Z. Wang, S. Liw, "A Band-Pass Sigma-Delta Modulator Employing Micro-Mechanical Resonator", IEEE Circuits and Systems, Vol. 1, Pp. 1-1041, May 2003

[4] M. Desvergne, C. Bernier, P. Vincent, Y. Deval, J-B. Begueret, "Intermediate Frequency Lamb Wave Resonators and Filters for RF Receiver Architectures", IEEE Electronics, Circuits and Systems, Vol. 46, No. 6, December 2006.

[5] A. Yahia, P. Benabes, "Bandpass Sigma-Delta Modulators Synthesis with High Loop Delay", IEEE International Symposium on Circuits and Systems, pp. 344-347, May 2001.

[6] O. Shoaei, W.M. Snelgrove, "A Multi-Feedback Design for LC BandPass Delta-Sigma Modulator", IEEE Circuits and Systems, Col. 1, Pp. 171-174, May 1995

[7] C. Banyasz, L. Keviczky, "A New Gap Metric for Robustness Measure and Regulator Design", Control and Automation, June 2001. 